Published in final edited form as:

Nano Lett. 2016 April 13; 16(4): 2556-2559. doi:10.1021/acs.nanolett.6b00108.

\title{
Super-Resolution Ultrasound Imaging in Vivo with Transient Laser-Activated Nanodroplets
}

\author{
Geoffrey P. Luke ${ }^{\dagger, \ddagger}$, Alexander S. Hannah ${ }^{\ddagger}$, and Stanislav Y. Emelianov ${ }^{\star}, \dagger, \ddagger$ \\ tDepartment of Electrical and Computer Engineering, The University of Texas at Austin, Austin, \\ Texas 78712, United States \\ ҒDepartment of Biomedical Engineering, The University of Texas at Austin, Austin, Texas 78712, \\ United States
}

\section{Abstract}

We have developed a method for super-resolution ultrasound imaging, which relies on a new class of blinking nanometer-size contrast agents: laser-activated nanodroplets (LANDs). The LANDs can be repeatedly optically triggered to undergo vaporization; the resulting spatially stationary, temporally transient microbubbles provide high ultrasound contrast for several to hundreds of milliseconds before recondensing to their native liquid nanodroplet state. By capturing high frame rate ultrasound images of blinking LANDs, we demonstrate the ability to detect individual recondensation events. Then we apply a newly developed super-resolution image processing algorithm to localize the LAND positions in vivo almost an order of magnitude better than conventional ultrasound imaging. These results pave the way for high resolution molecular imaging deep in tissue.

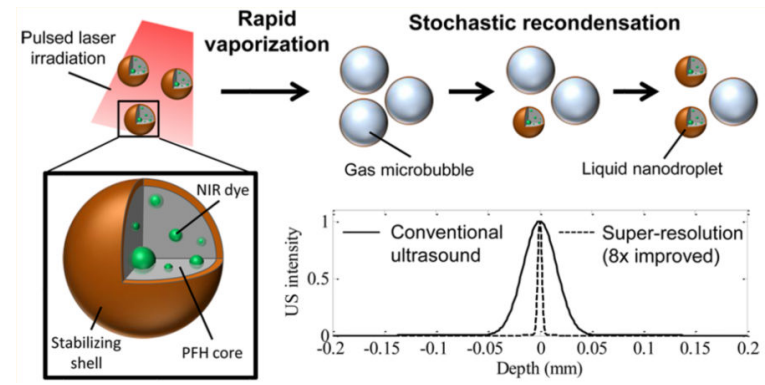

*Corresponding Author stas@gatech.edu.

Present Addresses

G.P.L.: Thayer School of Engineering, Dartmouth College, Hanover, NH 03755, USA.

A.S.H. and S.Y.E.: School of Electrical and Computer Engineering, Georgia Institute of Technology, Atlanta, GA 30332, USA, and The Wallace H. Coulter Department of Biomedical Engineering, Georgia Institute of Technology and Emory University School of Medicine, Atlanta, GA 30332, USA

The authors declare no competing financial interest.

ASSOCIATED CONTENT

Supporting Information

The Supporting Information is available free of charge on the ACS Publications website at DOI: 10.1021/acs.nanolett.6b00108.

Detailed methods, Figures S1-S4 (PDF)

Movie S1 (AVI) 


\section{Keywords}

Ultrasound; super resolution; perfluorocarbon nanodroplets; molecular imaging

Many super-resolution techniques in optical microscopy are founded on the principle that fluorescent molecules can be switched "on" or "off." If a sparse subset of all fluorescent molecules are either "on" (or undergo the transition) at a given time, then the signal generated from each single molecule can be fit to the point spread function (PSF) of the imaging system to localize the fluorophore's precise position. ${ }^{1-6}$ These techniques have demonstrated localization accuracy better than $10 \mathrm{~nm}$, which is greater than an order of magnitude improvement in spatial resolution. The primary limitation of optical imaging techniques, however, is that scattering in tissue prohibits high resolution imaging at depths greater than $1 \mathrm{~mm} .^{7}$

In contrast, the scattering of ultrasound waves in tissue is several orders of magnitude lower than optical scattering. ${ }^{8,9}$ This enables imaging resolution on the order of $100 \mu \mathrm{m}$ at depths of up to several centimeters. Because the contrast in ultrasound imaging is derived from variations in acoustic impedance, gaseous microbubbles are commonly used as ultra-sound contrast agents. ${ }^{10}$ Their relatively large size, however, restricts them to the vascular compartments. To address this, we and others have previously developed low-boiling-point liquid perfluorocarbon nanodroplets which can be triggered (e.g., via high intensity ultrasound or a pulsed laser) to undergo a phase change, generate a strong, one-time photoacoustic signal from vaporization, and create stable microbubbles for ultrasound contrast. ${ }^{1-14}$ Recently, nanodroplets containing a perfluorocarbon with a higher boiling point have been developed for their repeatable vaporization and sustained photo-acoustic signal. ${ }^{15,16}$

Here we present a new super-resolution technique for ultrasound imaging which uses high boiling point perfluorocarbon nanodroplets acting as "blinking" contrast agents. By capturing ultrasound images with a high frame rate, the signal from individual LANDs can be isolated, and their precise location can be estimated. This enables the ability to localize LANDs to within several micrometers and paves the way for high resolution molecular imaging at substantial depth in tissue.

In general, the LANDs consist of a perfluorohexane core (boiling point $=56^{\circ} \mathrm{C}$ ), a stabilizing shell (e.g., lipid, protein, or polymer), and an encapsulated photoabsorber to enable optical triggering (Figure 1a). For this study, we used a Zonyl FSO fluorosurfactant shell and a hydrophobic near-infrared dye (Epolight 3072). These LANDs have a mean diameter of $180 \mathrm{~nm}$ as measured by dynamic light scattering (Figure S1). Upon irradiation with a pulsed laser, a combination of localized heating and the resulting photoacoustic wave nucleates rapid vaporization of the perfluorohexane and the formation of a transient microbubble which persists for tens to hundreds of milliseconds, depending on the LAND composition, the environmental conditions, laser fluence, and incident ultrasound wave intensity (Figure 1b). This transient microbubble is visualized in ultrasound images as "blinking" with the "off" state corresponding to the liquid nanodroplet form and the "on" state corresponding to the gaseous microbubble form (Figure 1c). 
The recondensation of LANDs is a stochastic process, meaning that only a sparse subset of LANDs undergoes the gas-to-liquid transition at any given time. This process is likely driven by a combination of the laser energy, particle size, amount of encapsulated dye, ambient temperature, and local pressure. Thus, if the imaging frame rate is high enough (several hundred frames/second or greater), the response of individual LANDs can be isolated by taking the difference of adjacent frames (Figure 2a-c). After fitting the resulting image to a two-dimensional Gaussian approximation of the point spread function of the imaging system (Figure S3), the precise location of each LAND can be estimated as the position of the peak of the Gaussian fit (Figure 2d-e). It is important to note that the spacing between all particles need not be greater than the resolution of the imaging system; only the LANDs which undergo recondensation at a specific point in time must be separated accordingly. Indeed, several closely spaced particles can be localized within a single point spread function if they recondense at different times (Movie S1).

To demonstrate that the LANDs can be localized with subpixel resolution, we performed ultrasound imaging of a poly(acrylamide) tissue mimicking phantom with a low concentration (diluted $10000 \times$ from the as-prepared solution) of LANDs. In this case, the average spacing between LANDs was far greater than the resolution of the imaging system. Ultrasound imaging allows for detection sensitivity of single microbubbles (Figure S2). This enabled us to identify individual LANDs and track them over the course of multiple vaporization-recondensation events. Analysis of 9 LANDs over the course of 40 laser pulses showed that the estimated location of each LAND varied by much less than the innate resolution of the ultrasound imaging system. In fact, all estimations were within 8 and $20 \mu \mathrm{m}$ from the center of mass of all estimates in the axial and lateral directions, respectively (Figure 3a). The spatial distribution of points in Figure $2 \mathrm{f}$ is approximately Gaussian, and the full width half-maximum of the two-dimensional Gaussian describing the points was taken to be the resolution of the technique. In this case, a resolution of $4.6 \mu \mathrm{m}$ in the axial direction and $12.7 \mu \mathrm{m}$ in the lateral direction was achieved (Figure $3 \mathrm{~b}-\mathrm{c}$ ). This represents an 8 -fold improvement over the resolution of the ultrasound imaging system used in this study.

We demonstrated the application of this method in vivo by imaging the brain of a mouse which had undergone a craniotomy to create an acoustic window. The LANDs were systemically delivered to the vasculature via a retro-orbital injection. Ultrasound images were acquired in a single two-dimensional slice of the brain immediately following the injection (Figure 4). We clearly observed blinking pixels in the ultrasound images which directly correlated to the incident laser pulses; the blinking persisted for laser exposure times exceeding $60 \mathrm{~min}$. By isolating the blinking ultrasound signal, a high contrast map of the LANDs can be obtained (Figure 4a). The resolution of this image, however, is still limited by the diffraction of the acoustic waves and the bandwidth of the ultra-sound transducer. After applying our super-resolution method, much finer details emerge in the image (Figure $4 b)$. These features in the super-resolution likely correspond to micro-vasculature passing through the two-dimensional imaging plane. We evaluated the in vivo resolution by measuring the spread of points in two small features of the image (Figure 3c-d). In this case, we achieved a resolution of approximately $7-8 \mu \mathrm{m}$ in the axial direction and 14-16 $\mu \mathrm{m}$ in the lateral direction. These values are in close agreement with our in vitro studies. The slightly poorer resolution is likely due to minor physiological motion or phase aberrations. 
Stochasticity is required for resolving adjacent LANDs. This randomness can come either in the vaporization or in the recondensation process. We did observe instances where individual particles did not vaporize for a given laser pulse, indicating stochasticity of activation. More critical to this method, however, is the stochasticity associated with recondensation. One important factor in recondensation is the incident ultrasound energy. Higher transmitted ultrasound signals results in more rapid recondensation on average. Thus, it is possible that, as heat dissipates, the vaporized LANDs reach a supercooled state at which point the positive pressure of the imaging ultrasound wave nucleates recondensation. Since the imaging system and laser were not synchronized in this study, the exposure of LANDs to acoustic waves was not consistent from cycle-to-cycle. This could act as a significant driver in the stochasticity we observed. Other sources of stochasticity involve photobleaching of the encapsulated dye and splitting or coalescing of particles during vaporization. Finally, in the case of in vivo imaging of brain micovasculature, motion of the particles in circulation adds another level of stochasticity that would not be present with bound or immobile particles.

It is notable that these measurements do not rely on ballistic light and can be acquired in the diffuse regime of photon propagation. Thus, the developed technique has the potential to fill the gap between high-resolution but superficial optical imaging and other lower resolution deep penetrating biomedical imaging techniques. Furthermore, unlike previously developed super-resolution ultrasound imaging techniques, ${ }^{17-19}$ our method does not depend on microbubble motion. Therefore, these results could be extended to obtain high-resolution molecular images of molecularly targeted LANDs bound to cell-surface targets (Figure S4). Finally, the nanometer-sized LANDs have the potential to escape the vasculature via the enhanced permeability and retention effect for imaging of extravascular targets in cancer and other applications. Overall, the developed method could lead to imaging of cell-surface markers at near-single-cell resolution. This opens up a variety of exciting prospects in studying of tissue heterogeneity at small scales.

\section{Supplementary Material}

Refer to Web version on PubMed Central for supplementary material.

\section{ACKNOWLEDGMENTS}

We would like to thank Robin Hartman of The University of Texas at Austin for her assistance with the in vivo imaging study and Dr. Kimberly Homan of NanoHybrids Inc. for her help with the molecular targeting studies. This work was funded by the National Institutes of Health (grant R01EB008101) and the Breast Cancer Research Foundation.

\section{REFERENCES}

1. Betzig E, Patterson GH, Sougrat R, Lindwasser OW, Olenych S, Bonifacino JS, Davidson MW, Lippincott-Schwartz J, Hess HF. Science. 2006; 313:1642-1645. [PubMed: 16902090]

2. Bates M, Huang B, Dempsey GT, Zhuang X. Science. 2007; 317:1749-1753. [PubMed: 17702910]

3. Huang B, Wang W, Bates M, Zhuang X. Science. 2008; 319:810-813. [PubMed: 18174397]

4. Rust MJ, Bates M, Zhuang X. Nat. Methods. 2006; 3:793-796. [PubMed: 16896339]

5. Burnette DT, Sengupta P, Dai Y, Lippincott-Schwartz J, Kachar B. Proc. Natl. Acad. Sci. U. S. A. 2011; 108:21081-21086. [PubMed: 22167805] 
6. Wang Y, Fruhwirth G, Cai E, Ng T, Selvin PR. Nano Lett. 2013; 13:5233-5241. [PubMed: 24093439]

7. Ntziachristos V, Ripoll J, Wang LV, Weissleder R. Nat. Biotechnol. 2005; 23:313-320. [PubMed: 15765087]

8. d'Astous F, Foster F. Ultrasound Med. Biol. 1986; 12:795-808. [PubMed: 3541334]

9. Jacques SL. Phys. Med. Biol. 2013; 58:R37. [PubMed: 23666068]

10. Ferrara K, Pollard R, Borden M. Annu. Rev. Biomed. Eng. 2007; 9:415-47. [PubMed: 17651012]

11. Wilson K, Homan K, Emelianov S. Nat. Commun. 2012; 3:618. [PubMed: 22233628]

12. Hannah A, Luke G, Wilson K, Homan K, Emelianov S. ACS Nano. 2014; 8:250-259. [PubMed: 24303934]

13. Dayton PA, Zhao S, Bloch SH, Schumann P, Penrose K, Matsunaga TO, Zutshi R, Doinikov A, Ferrara KW. Mol. Imaging. 2006; 5:160. [PubMed: 16954031]

14. Sheeran PS, Luois S, Dayton PA, Matsunaga TO. Langmuir. 2011; 27:10412-10420. [PubMed: 21744860]

15. Asami, R.; Kawabata, K. IEEE Int. Ultrason. Symp. IEEE: pp; 2012. Repeatable vaporization of optically vaporizable perfluorocarbon droplets for photoacoustic contrast enhanced imaging; $\mathrm{p}$. 1200-1203.

16. Arnal B, Wei C-W, Perez C, Nguyen T-M, Lombardo M, Pelivanov I, Pozzo LD, O’Donnell M. Photoacoustics. 2015; 3:11-19. [PubMed: 25893170]

17. O'Reilly MA, Hynynen K. Med. Phys. 2013; 40:110701. [PubMed: 24320408]

18. Christensen-Jeffries K, Browning RJ, Tang M-X, Dunsby C, Eckersley RJ. IEEE Trans. Med. Imaging. 2015; 34:433-440. [PubMed: 25265604]

19. Errico C, Pierre J, Pezet S, Desailly Y, Lenkei Z, Couture O, Tanter M. Nature. 2015; 527:499_ 502. [PubMed: 26607546] 

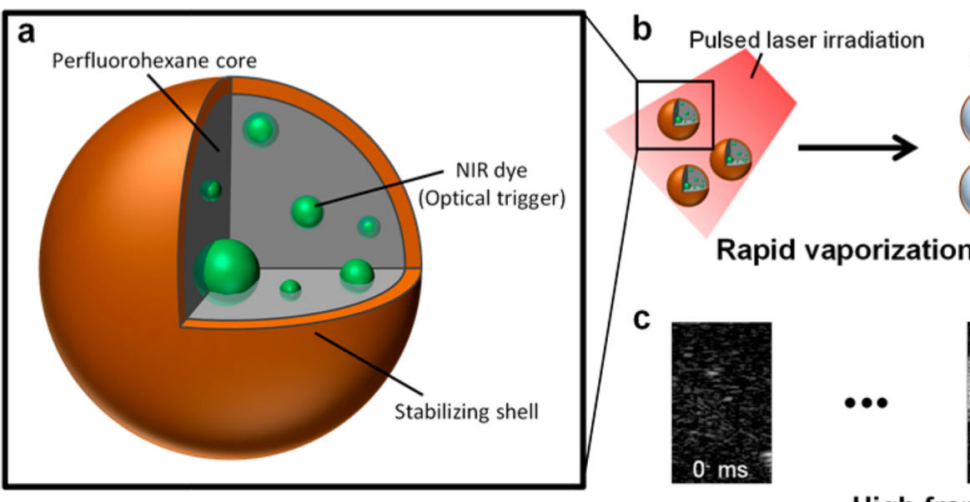

Gas microbubble
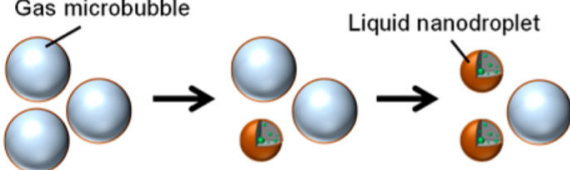

Figure 1.

(a) LANDs consist of a perfluorohexane core with encapsulated optical trigger (e.g., dye) and a stabilizing shell. (b) LANDs are vaporized by a pulsed laser irradiation (left). In their transient microbubble state, acoustic waves are reflected, providing ultrasound contrast. The LANDs recondense one at a time after the vaporization and no longer produce ultrasound contrast. (c) Ultrasound images before the laser trigger, immediately following the laser pulse, $30 \mathrm{~ms}$ after the laser pulse, and $60 \mathrm{~ms}$ after the laser pulse (from left to right) show the blinking behavior of LANDs. 

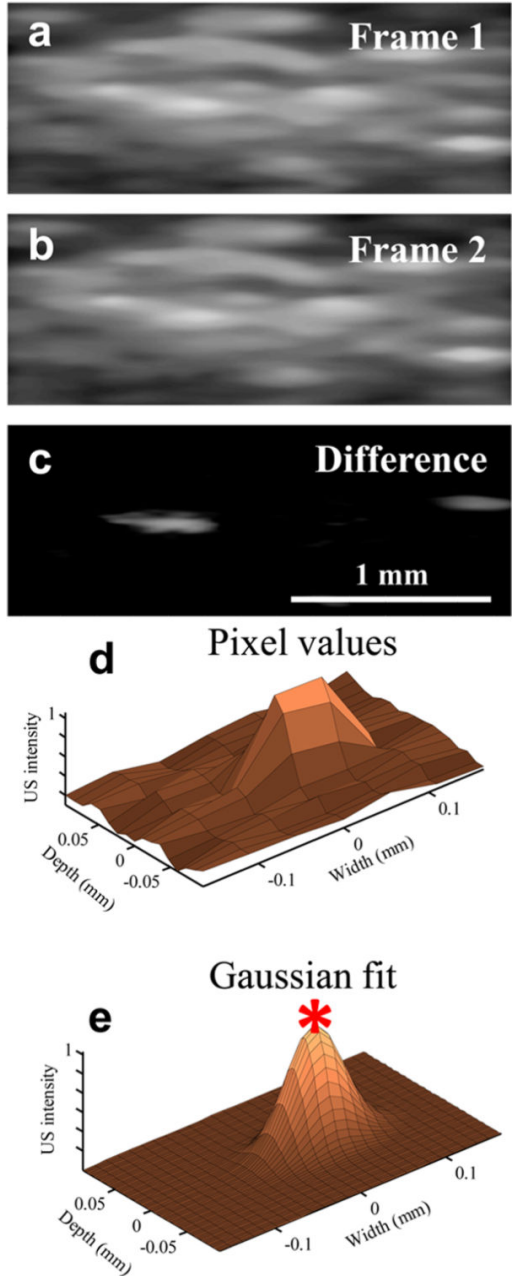

Figure 2.

(a-b) Two subsequent ultrasound frames and (c) their difference show the recondensation of two distinct LANDs. (d) The pixel values of a region of a difference image are approximated with (e) a 2-D Gaussian fit. The peak of the Gaussian is taken to be the exact location of the LAND. 

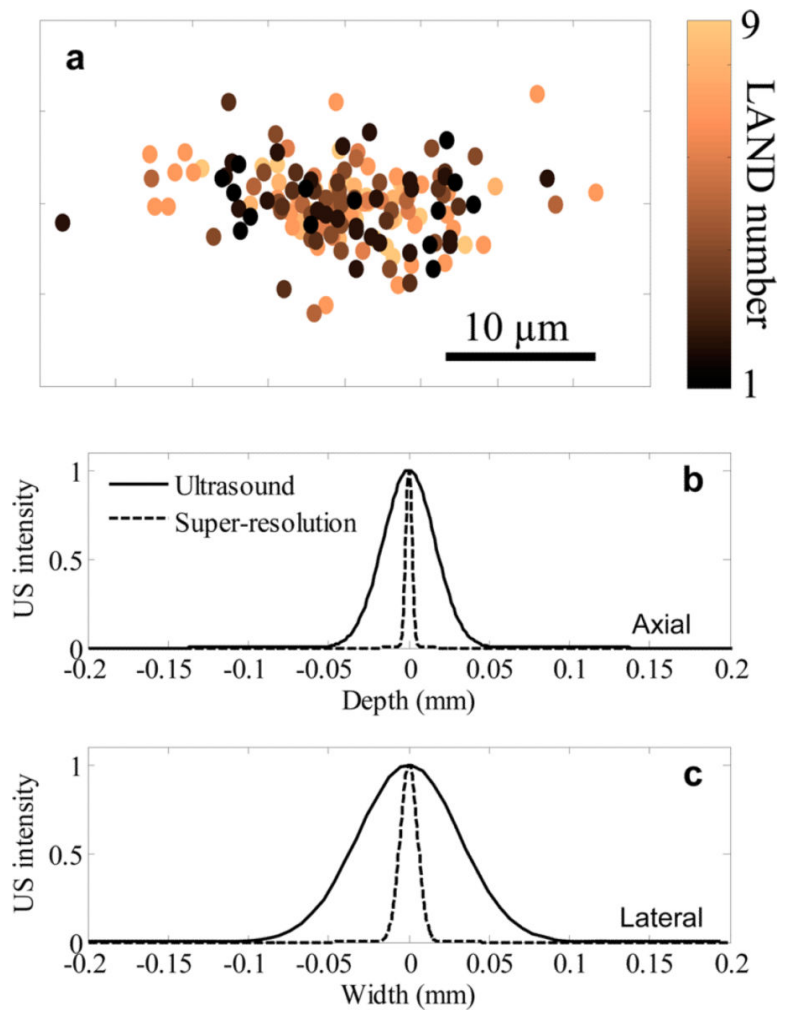

Figure 3.

(a) The centroid-subtracted locations of 9 LANDs repeatedly measured using multiple vaporization-recondensation cycles shows a narrow grouping of points. A Gaussian representing the data in (a) shows an 8-fold improvement in resolution in the (b) axial and (c) lateral dimensions. 

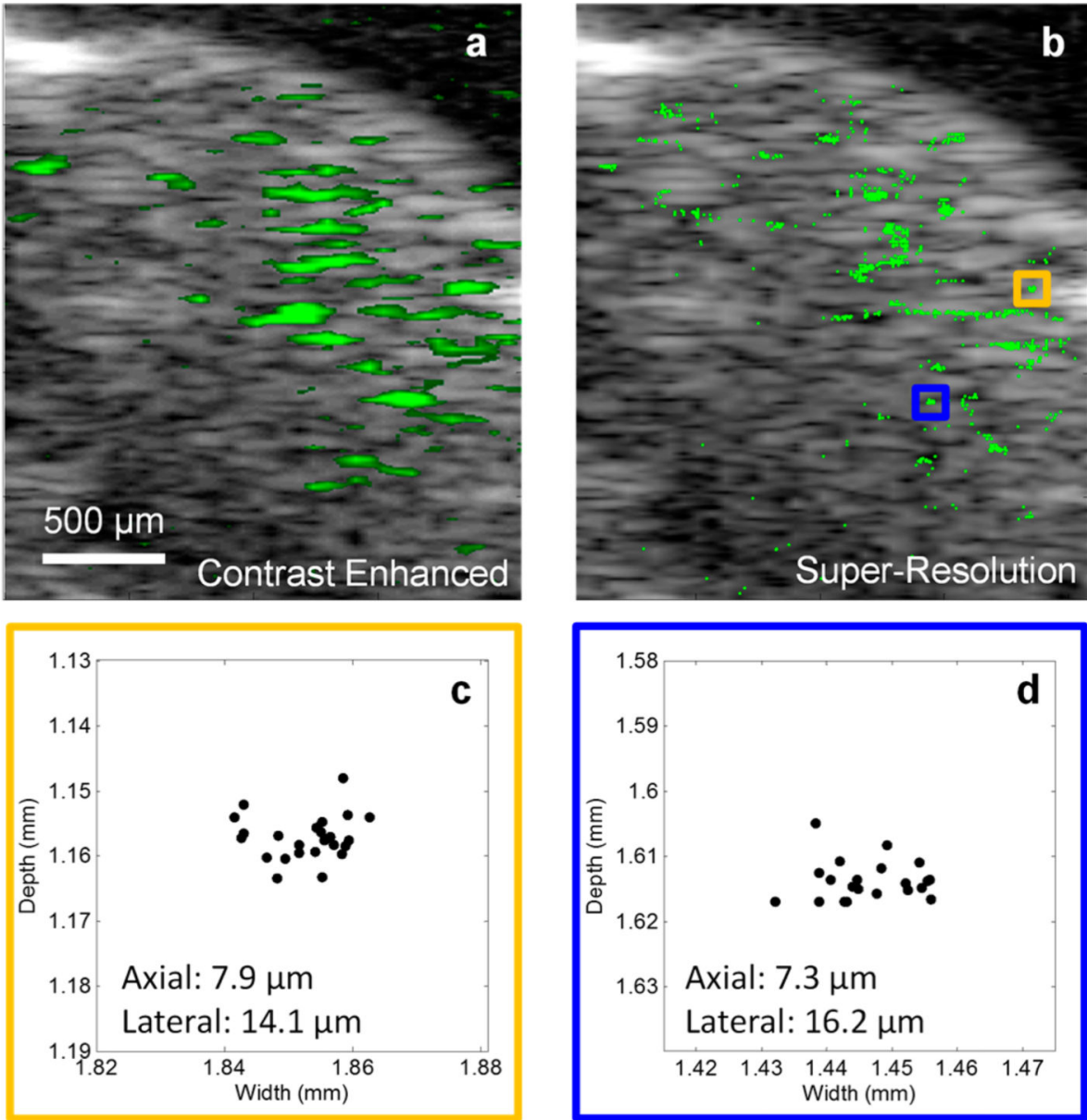

Figure 4.

(a) B-mode ultrasound imaging of a mouse brain (gray scale) with overlaid signal corresponding to blinking pixels (green). (b) After the super-resolution algorithm is applied to the images, the precise locations of the LANDs are determined, and finer details emerge. (c-d) Two small clusters of localized LANDs (corresponding to the orange and blue boxes in $b$, respectively) show that an axial resolution of $8 \mu \mathrm{m}$ and a lateral resolution of $16 \mu \mathrm{m}$ can be achieved in vivo. 